\title{
On dispersion relations and the statistical mechanics of Hawking radiation
}

\author{
Roberto Casadio* \\ Dipartimento di Fisica, Università di Bologna and \\ Istituto Nazionale di Fisica Nucleare, Sezione di Bologna, Italy
}

November 16, 2018

\begin{abstract}
We analyze the interplay between dispersion relations for the spectrum of Hawking quanta and the statistical mechanics of such a radiation. We first find the general relation between the occupation number density and the energy spectrum of Hawking quanta and then study several cases in details. We show that both the canonical and the microcanonical picture of the evaporation lead to the same linear dispersion relation for relatively large black holes. We also compute the occupation number obtained by instead assuming that the spectrum levels out (and eventually falls to zero) for very large momenta and show that the luminosity of black holes is not appreciably affected by the modified statistics.
\end{abstract}

\section{Introduction}

The gravitational collapse as described by general relativity can lead to the formation of space-times with peculiar causal structure and profound consequences on the (quantum) matter propagating on it. The most striking example is probably that, once the (apparent) horizon has formed, Hawking radiation [- [- generically sets off [2] and the mass of the source should then decrease by this quantum mechanical process. The main problem that remains with such a semiclassical picture is the determination of the backreacted metric and the corresponding time evolution of the source.

A different (but possibly related) problem is the role played by very high (trans-Planckian) frequencies. In fact, by tracking back in space a photon of frequency $\omega$ as measured by a distant observer, one immediately finds that its frequency is blue-shifted, in the optical approximation and neglecting the backreaction, according to the formula

$$
\omega^{*} \sim\left(1-\frac{R_{H}}{r_{e}}\right)^{-1 / 2} \omega
$$

where $r_{e}$ is the radial coordinate at the point of emission. It is clear that $\omega^{*}$ is unbounded from above if $r_{e}$ approaches the horizon radius $R_{H}$, and one is led to conclude that, in order to have $\omega$ finite, $\omega^{*}$ will soon exceed the Planck mass if $r_{e} \sim R_{H}$ as it is expected for Hawking quanta.

*casadio@bo.infn.it 
This is a very strong conclusion, since to study such energetic states one would need a full-fledged theory of quantum gravity.

More recently it was shown that, contrary to the above argument, trans-Planckian frequencies do not play a significant role in the Hawking process and the evaporation looks indeed insensitive to the presence of a UV (short distance) cut-off (for a review and list of references, see 33). This opens up the possibility that the spectrum of emitted quanta be not linear and a non-trivial dispersion relation avoid the production of trans-Planckian modes (see, e.g. Refs. [4, 5, 6] for interesting proposals). About the origin of the new dispersion relation little is known. One might argue that the blue-shift in Eq. (1.1) must be corrected for the true (backreacted) metric in the vicinity of the (apparent) horizon. In fact, since the Hawking quanta are produced inside the potential barrier that surrounds the horizon, a fraction of them gets trapped and forms a (thermal) bath which backreacts on the metric. Moreover, the quanta which escape through the barrier do not propagate in vacuum since they must cross such a bath [7]. The correct dispersion relation must then account for both aspects and, as such, reflects our inability to solve the main problem with black hole evaporation.

In the present paper we shall explore the connection between the dispersion relation and the statistical mechanics of Hawking quanta. We shall first work out general expressions in Section 2 which we then apply to both the canonical picture and the better sound microcanonical picture in Section 3. The starting point of the latter approach is the idea that black holes are (excitations of) extended objects ( $p$-branes), a gas of which satisfies the bootstrap condition [8, 9, 10]. This yields a picture in which a black hole and the particles it emits are of the same nature and the statistical mechanics of the radiation then follows straightforwardly from the area law of black hole degeneracy [11]. One obtains an improved law of black hole decay which is consistent with unitarity (energy conservation) and no information loss paradox is expected. In fact, black holes approximately decay exponentially in this picture, although departures from the canonical behavior occur only around (or below) the planck mass [10].

Of course, the statistical mechanical approach is global and does not allow us to fully determine the local behaviour of the fields (although some explicit connection with the dynamics of the local geometry and the backreaction can be drawn 12]). In particular, it yields the occupation number density of Hawking quanta, but one then needs an extra hypothesis to determine the wave modes that lead to such a density. In principle, the new wave modes should describe the propagation in the black hole metric with the backreaction included. An hypothesis of this sort was put forward in Ref. [9] and we shall show that quantitatively negligible corrections to the linear dispersion relation predicted by the canonical picture are required by the microcanonical treatment of the Hawking radiation, except that there is a natural cut-off at $\omega=M$, where $M$ is the black hole mass. This is practically ineffective for the problem of trans-Planckian frequencies since $M>1$ (in units of the Planck mass, with $c=\hbar=G=1$ ) for a (classical) black hole in four dimensions.

In the last part of the paper, Section \#, we shall reverse our line of reasoning and assume a dispersion relation in order to determine the corresponding occupation number and compare it with the canonical and microcanonical quantities. In particular, we shall choose a spectrum of the type proposed in Ref. [6] and show that it does not produce appreciable modifications to the luminosity of large black holes (in agreement with the general framework of Ref. [3]). In Section 5, we conclude by mentioning that this result might be significantly modified by the existence of extra dimensions [13], as the (microcanonical) luminosity was shown to depend strongly on the dimensionality of space-time [14]. 


\section{Occupation number density and wave modes}

An easy and instructive way of obtaining the standard (canonical) occupation number density of Hawking quanta is the following [15]. Consider a spherically symmetric four-dimensional metric in the Painlevé-Gulstrand form

$$
d s^{2}=-c^{2}(r, t) d t^{2}+[d r-v(r, t) d t]^{2}+r^{2} d \Omega^{2},
$$

where $d \Omega^{2}$ is the the line element of a unit two-sphere. The metric admits an (apparent) horizon if $r=R_{H}$ exists such that $v\left(R_{H}\right) \equiv v_{H}=-c_{H} \equiv-c\left(R_{H}\right)$. The surface gravity is then given by

$$
\kappa=\frac{g_{H}}{c_{H}}
$$

where

$$
\left.g_{H} \equiv \frac{1}{2} \frac{d\left(c^{2}-v^{2}\right)}{d r}\right|_{r=R_{H}}
$$

The wave modes

$$
\phi(r, t)=A(r, t) \exp [\varphi(r, t)]=A(r, t) \exp \left[i \omega t-i \int^{r} k\left(r^{\prime}\right) d r^{\prime}\right],
$$

solve the d'Alambertian equation in the eikonal approximation,

$$
\partial_{\mu} \varphi \partial^{\mu} \varphi+i \epsilon=0
$$

provided the wave-number is given by

$$
k=\frac{\omega}{\sigma(1+i \epsilon) c+v},
$$

where $\sigma=+1(-1)$ for outgoing (ingoing) modes.

Near the horizon $\left(r \sim R_{H}\right)$, ingoing modes $(\sigma=-1)$ have wave-number

$$
k_{\mathrm{in}} \approx-\frac{\omega}{2 c_{H}},
$$

and are defined for $r-R_{H}$ both positive and negative. Instead, purely outgoing modes $(\sigma=+1)$ exist only outside the horizon $\left(r>R_{H}\right)$ with

$$
k_{\mathrm{out}} \approx \frac{\omega}{\kappa\left(r-R_{H}\right)} .
$$

Additionally, in this set of coordinates one can consider "straddling" modes that are defined for

all values of $r>0$ and are swept "downstream" inside the horizon. For such modes one has (again for $r \sim R_{H}$ )

$$
\phi_{\text {straddle }}(r, t) \approx \exp \left\{i \omega t-i \frac{\omega}{\kappa} \ln \left|r-R_{H}\right|\right\}\left[\Theta\left(r-R_{H}\right)+\exp \left\{+\frac{\pi \omega}{\kappa}\right\} \Theta\left(R_{H}-r\right)\right],
$$


where the Boltzmann-like factor inside the square brackets emerges from the usual analyticity argument which relates ingoing and outgoing amplitudes [16] and corresponds to the inverse Hawking temperature

$$
\beta_{H}=\frac{2 \pi}{\kappa} .
$$

In fact, the physical vacuum is defined with respect to $\phi_{\text {straddle, }}$, since freely falling observers should not see any peculiarities as they cross the horizon. The Bogolubov coefficients of the transformation from the basis $\left\{\phi_{\text {straddle }}\right\}$ to the basis $\left\{\phi_{\text {in }}, \phi_{\text {out }}\right\}$ are then simply given by the (normalized) amplitude of the ingoing $\left(N_{\text {in }}\right)$ and outgoing $\left(N_{\text {out }}\right)$ parts of the "straddling" modes. From Eq. (2.9) one finds

$$
\left|N_{\text {in }}\right|^{2}=e^{\beta_{H} \omega}\left|N_{\text {out }}\right|^{2}
$$

The (wronskian) normalization condition

$$
\left|N_{\text {in }}\right|^{2}-\left|N_{\text {out }}\right|^{2}=1,
$$

then yields the thermal occupation number density

$$
n_{\beta}=\left|N_{\text {out }}\right|^{2}=\frac{1}{e^{\beta_{H} \omega}-1},
$$

for the outgoing Hawking quanta. To summarize, one has obtained the occupation number density as a consequence of the near horizon geometry.

By reversing the above argument, one could in principle assume a specific function for the occupation number density and then reconstruct the related possible metrics. If the exact $n$ were known, one would obtain some insight for the metric which takes the backreaction properly into account. It is therefore useful to rewrite some of the above expressions in terms of a (this far) unspecified function $n(\omega)$. In particular, by replacing $n_{\beta}$ with $n$ one obtains new Bogolubov coefficients such that

$$
\left|N_{\text {in }}\right|^{2}=e^{\ln \left[1+n^{-1}(\omega)\right]}\left|N_{\text {out }}\right|^{2}
$$

and the backreacted "straddling" modes are determined as

$$
\begin{aligned}
\phi_{\text {straddle }}(r, t) \approx & \exp \left\{i \omega t-\frac{i}{2 \pi} \ln \left[1+n^{-1}(\omega)\right] \ln \left|r-R_{H}\right|\right\} \\
& \times\left[\Theta\left(r-R_{H}\right)+\sqrt{1+n^{-1}(\omega)} \Theta\left(R_{H}-r\right)\right],
\end{aligned}
$$

again in the vicinity of the (apparent) horizon $\left(r \sim R_{H}\right)$.

\section{Canonical and microcanonical dispersion relations}

For the following, it is useful to introduce the dimensionless $\tilde{k} \equiv\left(r-R_{H}\right) k_{\text {out }}$. From Eq. (2.15) and any number density $n$ one finds that, for $r>R_{H}$,

$$
\tilde{k} \approx \ln \left[1+n^{-1}(\omega)\right] .
$$




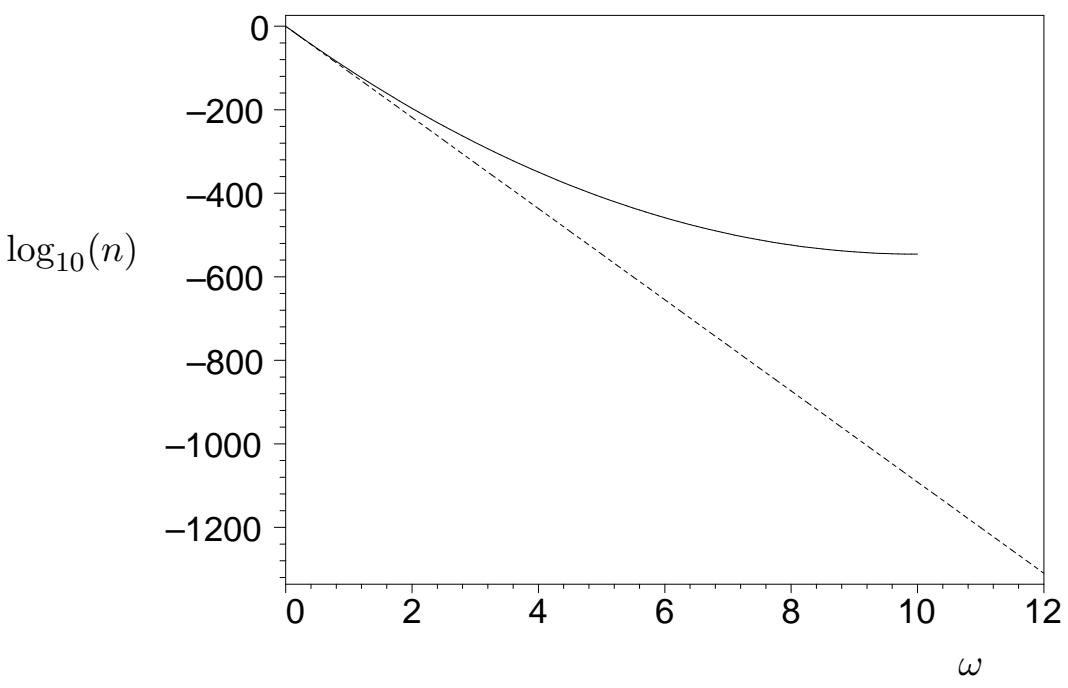

Figure 1: Behavior of $n_{M}$ (solid line) and $n_{\beta}$ (dotted line) for $M=10$. Note that $n_{M}=0$ for $\omega>M=10$ while $n_{\beta}$ is (formally) defined for all values of $\omega>0$.

This relation is in general difficult to invert, depending on the form of $n(\omega)$. For $n=n_{\beta}$, Eq. (3.1) reduces to $\tilde{k} \approx \beta_{H} \omega$ and, for fixed values of $r>R_{H}$, one finds

$$
\frac{d \omega}{d \tilde{k}} \approx \frac{1}{\beta_{H}}=\frac{1}{8 \pi M}
$$

We now recall that the occupation number density in the microcanonical ensemble is certainly a better approximation to the (unknown) exact expression [8]. It can be obtained directly from the area law without solving for the wave equation and is given by [8, 9]

$$
n_{M}= \begin{cases}C(\omega) \sum_{l=1}^{[[M / \omega]]} \frac{\exp \left[4 \pi(M-l \omega)^{2}\right]}{\exp \left(4 \pi M^{2}\right)} & \omega<M \\ 0 & \omega>M,\end{cases}
$$

where $M=1 / 4 \kappa$ is the (instantaneous) black hole mass and the function $C$ is a (unknown) factor which might account for high energy corrections coming, e.g., from string theory. In the following we shall set $C \sim 1$ unless differently specified. We also note that there is a natural cut-off at $\omega=M$ $(>1)$. For a comparison between $n_{M}$ and $n_{\beta}$ see Fig. 11.

For $n=n_{M}, \tilde{k}$ is a rather complicated function of $\omega$. However, one can make some approximations on considering that we are particularly interested in the high frequency regime, i.e., $\omega \sim 1$. For $M \gg 1$, it is useful to consider $M$ as a large integer and, for $1-\epsilon<\omega<1$ (with $0<\epsilon \ll 1$ ), one can easily compute

$$
\frac{d \omega}{d \tilde{k}} \approx-\frac{n_{M}\left(1+n_{M}\right)}{\omega\left(d n_{M} / d \omega\right)}
$$




\begin{tabular}{|c|c|c|c|c|}
\hline$M$ & $n_{M}$ & $\frac{d n_{M}}{d \omega}$ & $\frac{d \omega}{d \hat{k}}$ & $\frac{1}{8 \pi M}$ \\
\hline 1 & $3.5 \times 10^{-6}$ & $-4.4 \times 10^{-13}$ & $8 \times 10^{-2}$ & $4 \times 10^{-2}$ \\
\hline 10 & $2.0 \times 10^{-104}$ & $-4.5 \times 10^{-102}$ & $4 \times 10^{-3}$ & $4 \times 10^{-3}$ \\
\hline $10^{2}$ & $9.0 \times 10^{-1087}$ & $-2.3 \times 10^{-1083}$ & $4 \times 10^{-4}$ & $4 \times 10^{-4}$ \\
\hline $10^{3}$ & $2.8 \times 10^{-10910}$ & $-7.0 \times 10^{-10906}$ & $4 \times 10^{-5}$ & $4 \times 10^{-5}$ \\
\hline
\end{tabular}

Table 1: Occupation number density in the microcanonical picture for $\omega=1$ and comparison between microcanonical and canonical dispersion relations for $\omega \sim 1$ and various values of the black hole mass $M$.

In this range

$$
n_{M}=e^{-4 \pi M^{2}} \sum_{l=1}^{M} e^{4 \pi(M-l \omega)^{2}} \simeq e^{-8 \pi M \omega} \simeq n_{\beta}
$$

and

$$
\frac{d n_{M}}{d \omega}=e^{-4 \pi M^{2}} \sum_{l=1}^{M} 8 \pi(l \omega-M) e^{4 \pi(M-l \omega)^{2}} \simeq-8 \pi M e^{-8 \pi M \omega} \simeq \frac{d n_{\beta}}{d \omega}
$$

from which

$$
\left.\frac{d \omega}{d \tilde{k}}\right|_{\omega \sim 1} \approx \frac{1}{8 \pi M}
$$

in agreement with Eq. (3.2). The above turns out to be a rather good estimate for $M \sim 10$ and greater, as one can check numerically (see table 1).

For small values of $M(\sim 1)$, one must properly take into account the finite sum appearing in Eq. (3.3). The result of a numerical analysis is shown in Fig. 2 for $M=1$ and in Fig. 3 for $M=10$. As was expected from the figures given in Table 1, the dispersion relation for $M=10$ is visibly linear and does therefore not differ from the canonical picture. For $M=1$ the curve departs from linearity and turns upward for $\omega$ approaching the Planck energy.

\section{Occupation number from dispersion relations}

Upon solving Eq. (3.1) for $n(\omega)$ one obtains

$$
n=\frac{1}{e^{\tilde{k}}-1},
$$

which is uniquely defined only for intervals of $\omega$ where the function $\tilde{k}(\omega)$ is single valued. This seems to be true for the two cases inspected in the previous Section, and would also hold for a spectrum which goes asymptotically constant for large $\tilde{k}$ [ [ Epstein functions suggested in Ref. [6]. The latter can be considered as an extension to all values of $\tilde{k}$ of the spectrum studied in Ref. [5],

$$
\omega^{2}=\frac{\tilde{k}^{2}}{k_{0}^{2}}\left(1-\frac{\tilde{k}^{2}}{\Lambda^{2}}\right)
$$




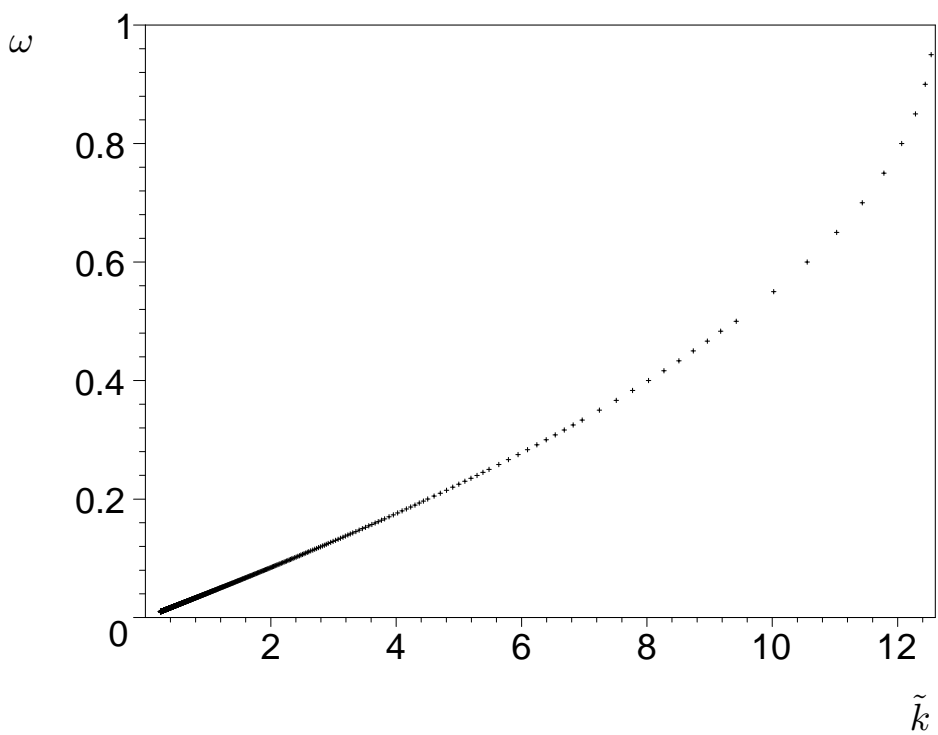

Figure 2: Microcanonical dispersion relation for $M=1$.

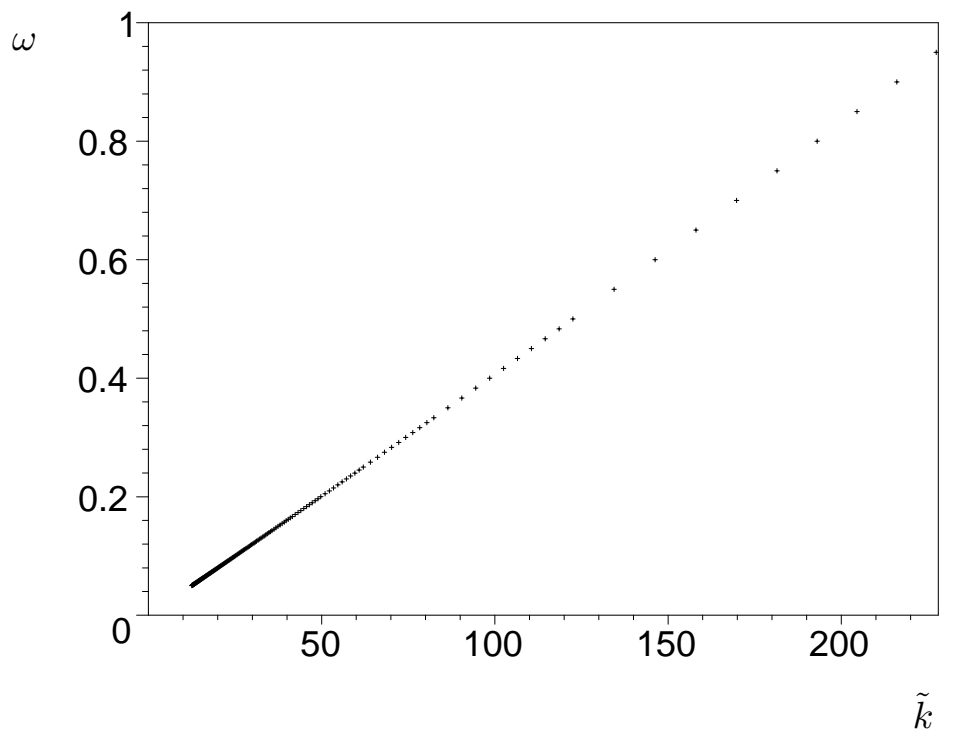

Figure 3: Microcanonical dispersion relation for $M=10$. 
which is defined only for $\tilde{k}<\Lambda$ and is presumably meaningful only as the next-to-leading order expansion at small $\tilde{k}$ of the correct dispersion relation.

We shall here consider a particular case of the family of functions studied in Ref. [6], namely

$$
\omega^{2}=\frac{\tilde{k}^{2}}{k_{0}^{2}}\left[\frac{\epsilon}{1+e^{\tilde{k} / k_{C}}}+\frac{(4-2 \epsilon) e^{\tilde{k} / k_{C}}}{\left(1+e^{\tilde{k} / k_{C}}\right)^{2}}\right],
$$

where $k_{C}$ determines the location of the maximum of $\omega$. We also demand that $n(\omega) \simeq n_{\beta}(\omega)$ for $\omega \ll 1$. This uniquely determines the coefficients $k_{0}=\beta_{H}$ and $\epsilon=0$ from equating the two lowest order coefficients in the Taylor expansion of Eq. (4.3) near $\tilde{k}=0$ to the right hand side of Eq. (3.2). The constant $k_{C}$ can be fixed by requiring that the maximum of $\omega$ is close to 1 . Since the derivative of $\omega(\tilde{k})$ with respect to $\tilde{k}$ vanishes for $\tilde{k}=k_{m} \simeq(5 / 2) k_{C}$, from $\omega\left(k_{m}\right)=1$ one obtains $k_{C} \simeq(3 / 4) \beta_{H}$ and $k_{m} \simeq(15 / 8) \beta_{H}$. Finally,

$$
\omega \simeq \frac{\tilde{k}}{\beta_{H}} \operatorname{sech}\left(\frac{2 \tilde{k}}{3 \beta_{H}}\right) .
$$

In Fig. A we plot a comparison between the dispersion relation (4.4) and the canonical dispersion relation (3.2) for $M=10$ (we recall that the same dispersion relation follows from the microcanonical picture for such a large mass as shown in Table 1 and Fig. 3). In Fig. 5 we then display a comparison between the corresponding occupation number densities $n$ and $n_{\beta}$. We note that in the range $0<\omega<1, n_{M} \geq n_{\beta}$ while $n \leq n_{\beta}$. One might from this infer that the microcanonical number density must be corrected for $\omega \sim 1$ by a suitable $C(\omega)$ in Eq. (3.3).

Having determined a novel occupation number density, we can now proceed to estimate the corresponding luminosity for an evaporating black hole, which can be formally written as [1]

$$
\mathcal{L}=\mathcal{A} \int_{0}^{\infty} \Gamma(\omega) n(\omega) \omega^{3} d \omega
$$

where $\Gamma \sim 1$ is the grey-body factor and $\mathcal{A}=16 \pi M^{2}$ the horizon area. We now note that, since $k_{m} \gg 1$ for a black hole of mass $M>1$, the number density for momenta $\tilde{k}>k_{m}$ is highly suppressed by the form of Eq. (4.1). Further, $\omega(\tilde{k})$ vanishes exponentially for $\tilde{k} \gg k_{m}$. One can therefore neglect the contribution of such modes, use the number density in Eq. (4.1) in the range $0<\tilde{k}<k_{m}$ and approximate the luminosity as

$$
\begin{aligned}
\mathcal{L} & \simeq \frac{\beta_{H}^{2}}{4} \int_{0}^{1} n(\omega) \omega^{3} d \omega \\
& \simeq \frac{1}{4 \beta_{H}^{2}} \int_{0}^{k_{m}} \operatorname{sech}^{4}\left(\frac{2 \tilde{k}}{3 \beta_{H}}\right)\left[1-\frac{2 \tilde{k}}{3 \beta_{H}} \tanh \left(\frac{2 \tilde{k}}{3 \beta_{H}}\right)\right] \frac{\tilde{k}^{3} d \tilde{k}}{e^{\tilde{k}}-1} \\
& \sim M^{-2},
\end{aligned}
$$

where $k_{m}$ is the value of $\tilde{k}$ at which the term in square brackets (proportional to $d \omega / d \tilde{k}$ ) vanishes and the last line follows from dimensional analysis. The above Eq. (4.5) is just the standard canonical result [1]. The integral can also be estimated more precisely by changing the integration variable,

$$
\mathcal{L}=\frac{\beta_{H}^{2}}{4} \int_{0}^{x_{m}} \operatorname{sech}^{4}(x)[1-x \tanh (x)] \frac{x^{3} d x}{e^{\frac{3}{2} \beta_{H} x}-1},
$$




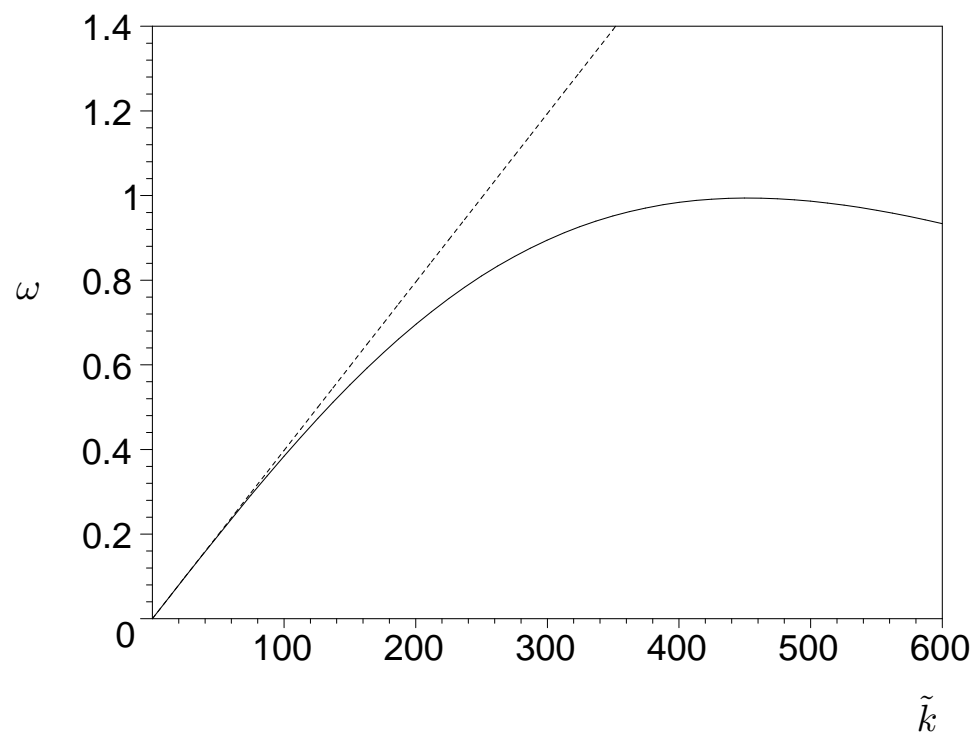

Figure 4: Comparison between the dispersion relation (4.4) (solid line) and the canonical dispersion relation (3.2) (dotted line) for $M=10$.

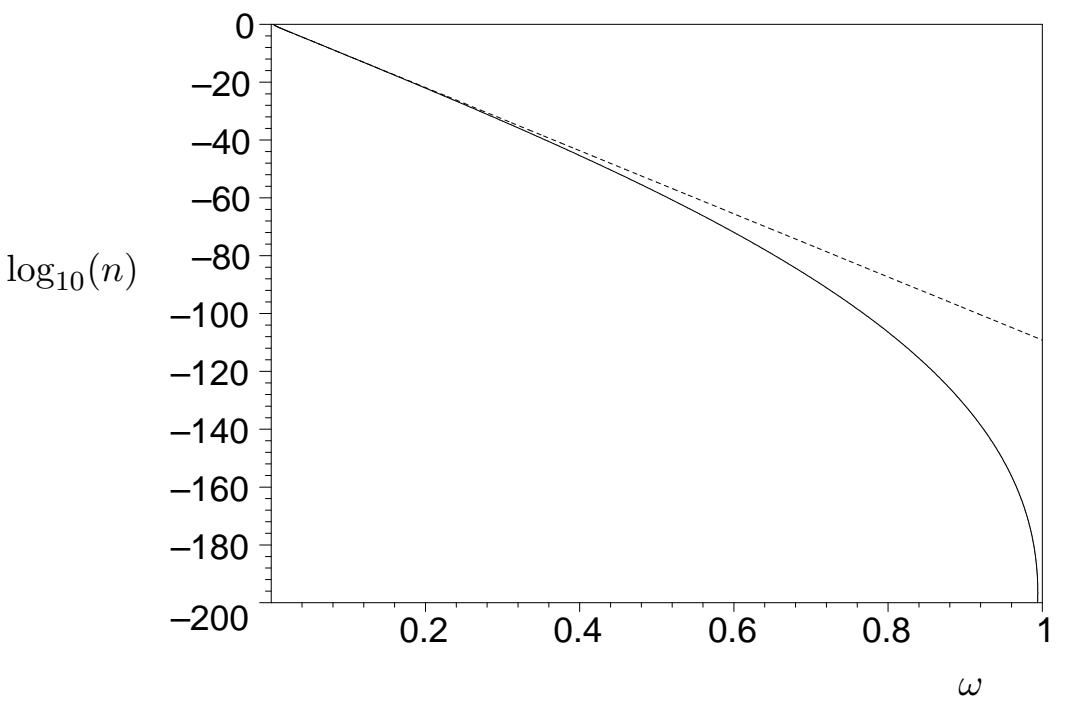

Figure 5: Behavior of the occupation number density $n$ (solid line) corresponding to the dispersion relation (4.4) compared with $n_{\beta}$ (dotted line) for $M=10$. 


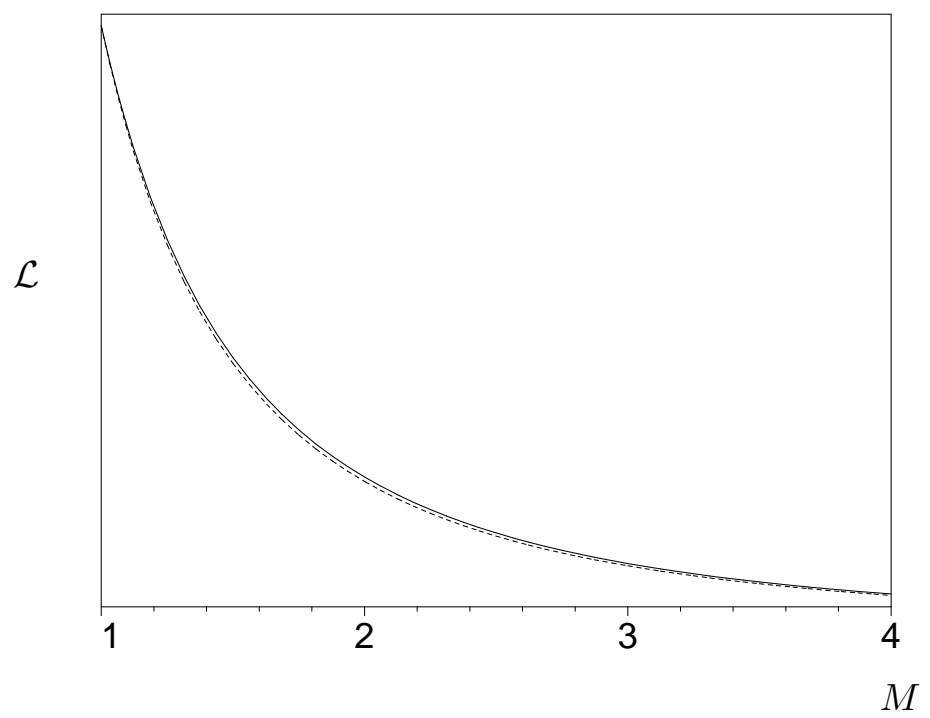

Figure 6: Black hole luminosity determined by the Epstein dispersion relation (4.4) (solid line) compared with the canonical luminosity (dotted line). Vertical units are arbitrary.

where $x_{m} \equiv x\left(k_{m}\right) \simeq 1.2$, and performing the integration numerically. The result is displayed for comparison with the canonical luminosity in Fig. 6 which shows that Eq. (4.6) is indeed correct to an exceedingly good approximation. We finally note that, since the microcanonical luminosity does not significantly differ from the canonical expression for four-dimensional black holes (necessarily with $M>1$ [10]), the luminosity (4.6) computed in this section is also in agreement with the microcanonical result.

\section{Conclusions and outlook}

In this paper we have studied how the statistical mechanics of the black hole evaporation is affected by the high energy behavior of Hawking quanta in four dimensions. We have found that one can consider large deviations from a linear dispersion relation at near Planckian frequencies without changing the luminosity of a black hole. In particular, the fact that the new dispersion relations advocated in Ref. [6] do not change the luminosity of a black hole with $M>1$ is a direct verification of the general framework described in Ref. [3]. We have, however, not attempted at any estimate of how such modifications affect the laws of black hole thermodynamics, nor whether they can be indeed derived from a fundamental theory.

Although we have not explicitely considered black holes lighter than the Planck mass in this paper, we suspect one would obtain different results for those cases [10]. Such objects are outside the domain of classical general relativity in four dimensions, since their Compton wavelength would be larger then the horizon radius. However, with more than four available dimensions [13] black holes could exist with $M_{0}<M<1$ (where $M_{0}$ is of the order of the fundamental mass scale of gravity). Further, the scale below which microcanonical corrections to the luminosity become 
significant in that scenario is given by the critical mass $M_{c}$ (much larger then the Planck mass) above which the black hole starts to behave like a purely four-dimensional object [14]. It then follows that for black holes with $M_{0}<M<M_{c}$ one expects that modification of the dispersion relation for frequency $1<\omega<M_{c}$ indeed affects the luminosity. We hope to extend our analysis along this line in a future publication.

\section{Acknowledgement}

I would like to thank M. Bastero-Gil and L. Mersini for useful discussions and B. Harms for reading the manuscript.

\section{References}

[1] S.W. Hawking, Nature 248, 30 (1974); Comm. Math. Phys. 43, 199 (1975).

[2] P. Hajicek, Phys. Rev. D 36 (1987) 1065.

[3] T. Jacobson, Prog. Theor. Phys. Suppl. 136 (1999) 1.

[4] W. Unruh, Phys. Rev. D 51 (1995) 2827.

[5] S. Corley and T. Jacobson, Phys. Rev. D 54 (1996) 1568

[6] M. Bastero-Gil, What can we learn by probing trans-Planckian physics, hep-ph/0106133;

L. Mersini, Dark energy from the transplanckian physics, hep-ph/0106134;

L. Mersini, M. Bastero-Gil and P. Kanti, Phys. Rev. D 64 (2001) 043508.

[7] R. Parentani, Phys. Rev. D 63 (2001) 041503.

[8] B. Harms and Y. Leblanc, Phys. Rev. D 46 (1992) 2334; Phys. Rev. D 47 (1993) 2438; Ann. Phys. 244 (1995) 262; Ann. Phys. 244 (1995) 272; Europhys. Lett. 27 (1994) 557; Ann. Phys. 242 (1995) 265;

P.H. Cox, B. Harms and Y. Leblanc, Europhys. Lett. 26 (1994) 321;

R. Casadio, B. Harms and Y. Leblanc, Phys. Rev. D 571309 (1998).

[9] R. Casadio and B. Harms, Phys. Rev. D 58 (1998) 044014.

[10] R. Casadio and B. Harms, Mod. Phys. Lett. A17 (1999) 1089.

[11] J.D. Bekenstein, Lett. Nuovo Cim. 4 (1972) 737; Phys. Rev. D 7 (1973) 2333.

[12] R. Casadio, Phys. Lett. B 511 (2001) 285.

[13] N. Arkani-Hamed, S. Dimopoulos and G. Dvali, Phys. Lett. B 429, 263 (1998); Phys. Rev. D 59, 0806004 (1999);

I. Antoniadis, N. Arkani-Hamed, S. Dimopoulos and G. Dvali, Phys. Lett. B 436, 257 (1998);

L. Randall and R. Sundrum, Phys. Rev. Lett. 83, 4690 (1999).

[14] R. Casadio and B. Harms, Phys. Rev. D 64 (2001) 024016; Can black holes and naked singularities be detected in accelerators?, hep-th/0110255 
[15] M. Visser, Essential and inessential features of Hawking radiation, hep-th/0106111.

[16] N.D. Birrell and P.C.W. Davis, Quantum fields in curved space (Cambridge, Cambridge University Press, 1982). 